\title{
Simulation of Class E Inverter Based Induction Heater Using Simulink
}

\author{
S.Arumugam and S.Ramareddy
}

\begin{abstract}
This paper presents simulation of class E inverter based induction heater system using simulink. DC is converted into high frequency $\mathrm{AC}$ using class $\mathrm{E}$ inverter. This high frequency $\mathrm{AC}$ is used for induction heating .Open and closed loop systems are modeled and they are simulated using Mat lab Simulink.The results of open and closed loop system are presented.
\end{abstract}

\section{INTRODUCTION}

IN the high-efficiency Class-E power amplifier, the transistor is used as a switch. The resonator $\mathrm{L}_{0}, \mathrm{C}_{0}$ is used to block the harmonic frequencies and DC component, forcing the output current $I_{0}$ to approximate a sine wave at the fundamental frequency, with harmonic content as discussed in [10]. The radio frequency choke $L_{R F}$ is assumed to be ideal such that it conducts only the DC current. The current into switch $\mathrm{S}$ and capacitor $\mathrm{C}_{\mathrm{s}}$ must be a DC -offset sine wave, with some harmonic content as discussed in [10]. By appropriately adjusting the amplitude and phase of the load current, a solution is found with zero capacitor charge just prior to turn-on.

This results in a switching waveform with zero voltage and zero voltage slope at turn-on. The conditions are those of the well-known Class-E switching [2], [11], [12]. This allows high-efficiency operation at frequencies up to 10 $\mathrm{GHz}$. Additionally, the Class-E topology can be implemented with fewer components because the power MOSFETs' parasitic capacitors can be incorporated into the circuit. These benefits have allowed the Class-E topology to achieve high power density, thus reducing the size and weight of the equipment. However, a blocking filter $\mathrm{L}_{0}, \mathrm{C}_{0}$ is needed to block the harmonic frequencies The shrinking size of electronic equipment demands ever-increasing power densities at high switching frequencies and a minimal parts count for the circuit technology. In an attempt to minimize the parts count with Class-E operation, the oneinductor one-capacitor Class-E high-efficiency switchingmode tuned PA [1] provides a more simplified circuit. Nevertheless, this simplified single-ended circuit is appropriate only for applications in which the harmonic content and the phase-modulation noise of the output are not important criteria. It is therefore desirable to retain the functions of the conventional Class-E features; i.e., that the amplifier can be operated with high efficiency at very high frequencies and provides a sinusoidal output waveform and power-handling capability without increasing the complexity of the power circuits.

\footnotetext{
S.Arumugam, Bharath University, Chennai, India, s_arumugam@rediffmail.com srr_victory@yahoo.com
}

The proposed push-pull Class-E amplifier and the conventional single-ended circuit configuration that includes one inductor and one capacitor. As expected, the harmonic contents of output voltage are significantly reduced in the proposed push-pull amplifier. However, the amplitudes of the positive and negative half-cycle in the output-voltage waveform are not symmetrical, which may cause a small second-harmonic component

Inductors and capacitors are not identical, Because of their nonlinearity and that the tolerance of the component characteristics differ appreciably, the appearance of even harmonics must be expected. The approaches presented here can be applied to the analysis and design of other Class-E amplifier configurations or with more complicated circuits in exact designs. Further, it should be noted that for this topology, the circuit described in this paper has two operational points that are performed by the ZVZS and ZVZC switching. Unlike the single-ended Class-E amplifier [1], the push-pull architecture is able to achieve a sinusoidal output waveform and high power-handing capability. For instance, a symmetrically driven push-pull Class-E amplifier has been proposed for high-power applications as shown in Fig. 1

With the symmetrical gate-driving signals, theoretically, the even harmonics are entirely cancelled at the load, and thus there are fewer harmonic distortions (HDs). However, this doubled parts-count configuration incurs penalties on the overall efficiency and the design cost. Recently, the Class-E/F [16] and the current-mode Class-D [17], with low peak voltage and/or low rms current, have been implemented as a high-frequency amplifier, However, the current-mode Class-D and the Class-E/F only achieve zerovoltage switching (ZVS) conditions. Fortunately, there is a more elegant way to further reduce the switching loss, if the switch current increase gradually from zero after the switch is closed. This paper suggests a push-pull Class-E resonant PA with a simple LC load network and a load resistor $\mathrm{R}_{\mathrm{L}}$ in each half-amplifier, An overlapped capacitor-voltage waveform is utilized to achieve the nominal Class-E conditions without increasing the complexity of the power circuits. For nominal operation, the following performance parameters are determined: the current and voltage waveforms, the peak values of drain current and drain-tosource voltage, the output power, the power-output capability, and the component values of the load network [18].

\section{PRINCIPLE OF OPERATION}

The basic schematic of the proposed push-pull Class-E series- parallel LCR resonant PA is shown in Fig. 2. It contains two MOSFETs, two inductors, two capacitors, and 
a load resistance.

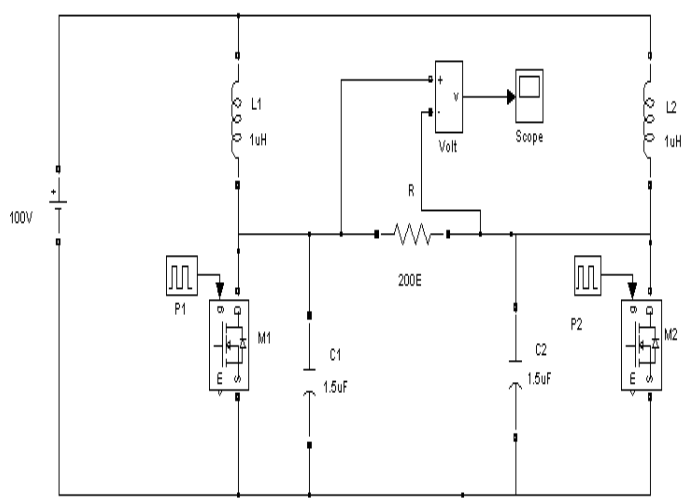

Fig 2.b.Proposed Simulation model

Switches S1 and S2 are complementarily activated to drive periodically at the operating frequency $f=\omega / 2 \Pi$ as in a push-pull switching PA [27], i.e., the switch waveforms are identical, except that the phase shifts between S1 and S2 are $\Pi$ with an "on" duty ratio $D$ of less than $50 \%$. The simplest type of half-amplifier, as shown in Fig. 1(d), is a series-parallel resonant circuit, which consists of an inductor $\mathrm{L}$ in series with a paralleled capacitor $\mathrm{C}$ and resistor $R$. The resistor $R_{L}$ is the load to which the AC power is to be delivered, with neither end connected to a ground. It is suitable for a load that is balanced to a ground, but most RF-power loads have one end connected to a ground.

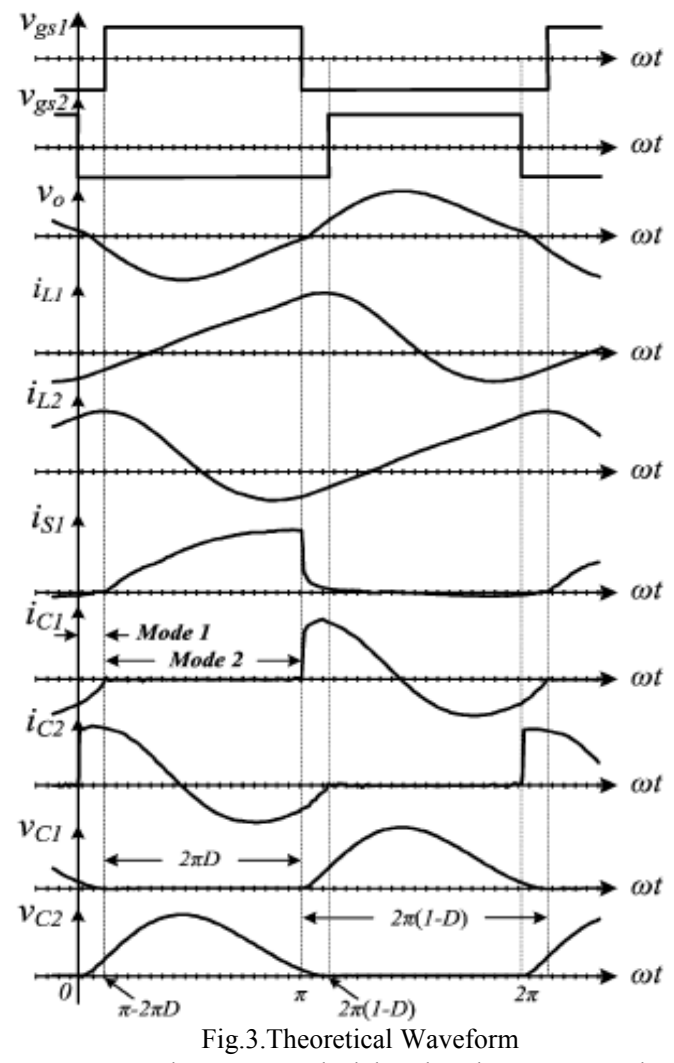

To accommodate grounded loads, the proposed topology needs to add one of the following: a balun that can be used to provide the interface with the amplifier [28]; or a twowinding transformer (that has $\mathrm{V}_{\mathrm{i}}$ connected to a center-tap on the primary winding), between the grounded load (on the grounded secondary winding) and the drains of S1 and S2 (connected to the ends of the center-tapped primary winding). The switching sequences and theoretical waveforms for the steady-state operation of the proposed amplifier are illustrated in Fig. 3. To reduce the transistor turn-on power losses, the switch current $i_{s}$ increase gradually from zero after the switch is closed. The proposed push-pull Class-E PA uses a pair of LC resonant networks with an overlapped capacitor-voltage waveform; this offers additional degrees of freedom, and thus there are two operational points that can validly achieve this situation:

Case 1) [Zero-Voltage Zero-Slope Switching (ZVZSS)]: In this case, the nominal operating conditions of ZVS and zero-voltage-slope switching (ZVSS) are simultaneously satisfied. Namely

$$
\left\{\begin{array}{c}
v_{C 1}(\pi-2 \pi \mathrm{D})=0 \\
\frac{\operatorname{dvC} 1(\pi-2 \pi \mathrm{D})}{d t}=0
\end{array}\right.
$$

Case 2) [Zero-Voltage Zero-Current Switching (ZVZCS)]:

The operation principle in the commutation of this case is solved by the following simultaneous equations:

$$
\left\{\begin{aligned}
& v_{C 1}(\pi-2 \pi D)=0 \\
& i_{L 1}(\pi-2 \pi D)=-v_{C 2}(\pi-2 \pi D) \\
& \mathrm{R}_{\mathrm{L}}
\end{aligned}\right.
$$

In order to satisfy both case 1 and case 2 , it is necessary to find the current $i_{L 1}=-i_{R L}$ by which the switch current increases gradually from zero at time $\mathrm{t}=(\Pi-2 \Pi \mathrm{D}) / \omega$, asshown in Figs. 2 and 3. The duty ratio must be kept at less than $50 \%$ so that the capacitor-voltage waveforms $\mathrm{V}_{\mathrm{Cl}}$ and $\mathrm{V}_{\mathrm{c} 2}$ can be overlapped.

\section{SimULATION RESULTS}

Class E inverter system is simulated using simulink and the results are given here.

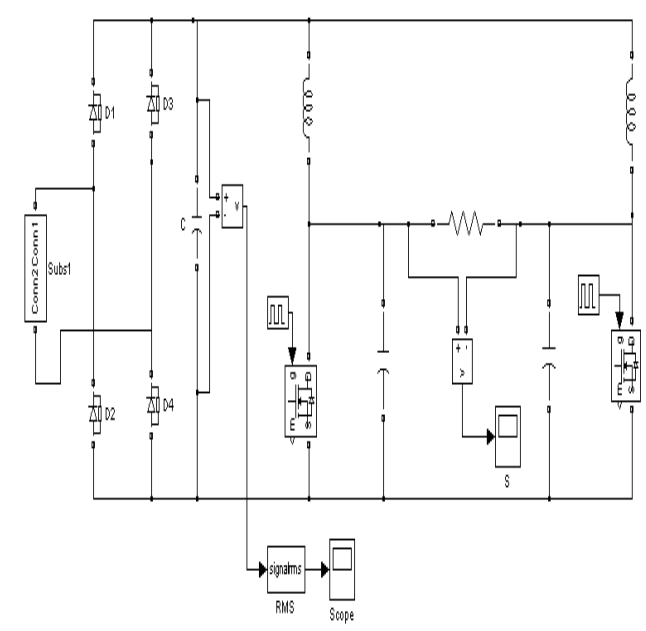

Fig.4a. Matlab Simulation circuit

Class E inverter circuit is shown in Fig 4a. DC input voltage is shown in Fig 4b. Driving pulses are shown in Fig 
4c.
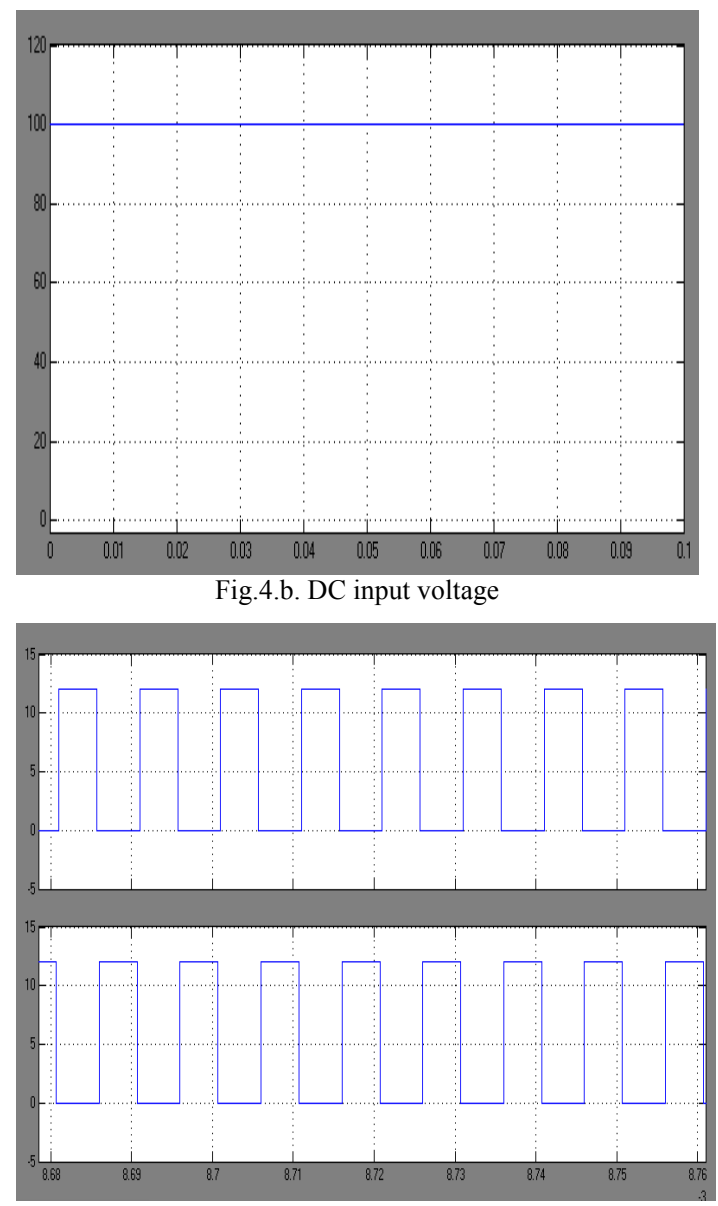

Fig.4.c.Driving pulses

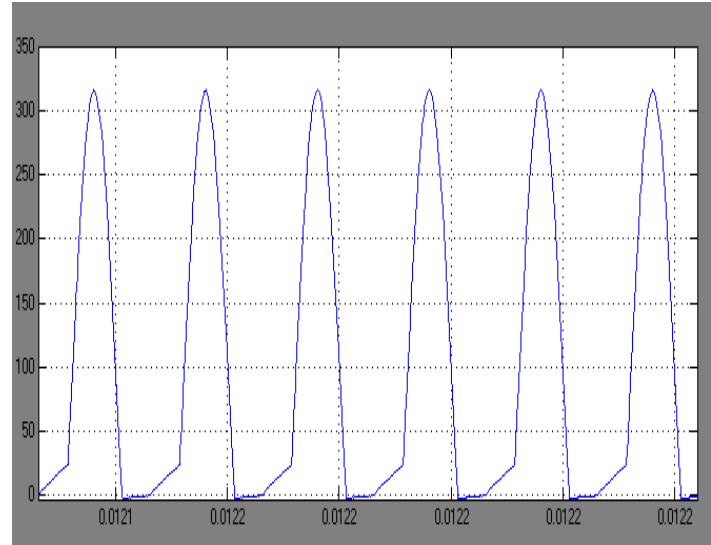

Fig $4 . d$ voltage across switch 1

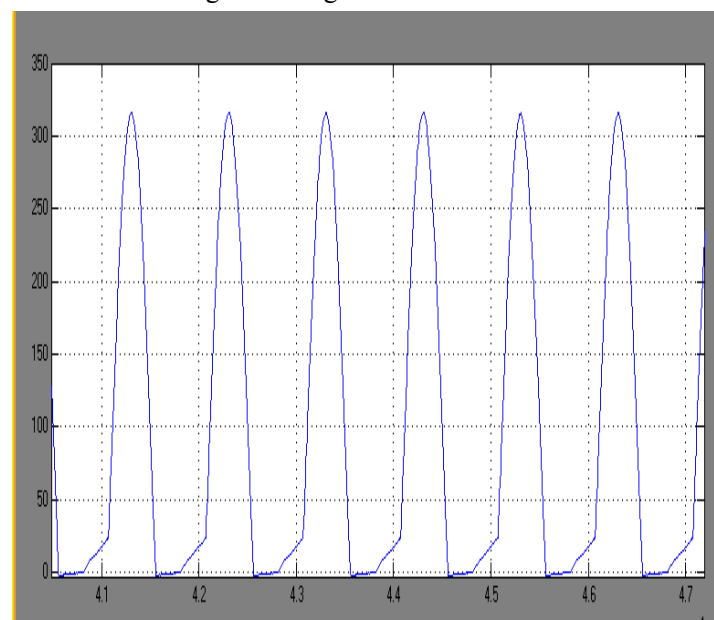

Fig 4.eVoltage across Switch 2
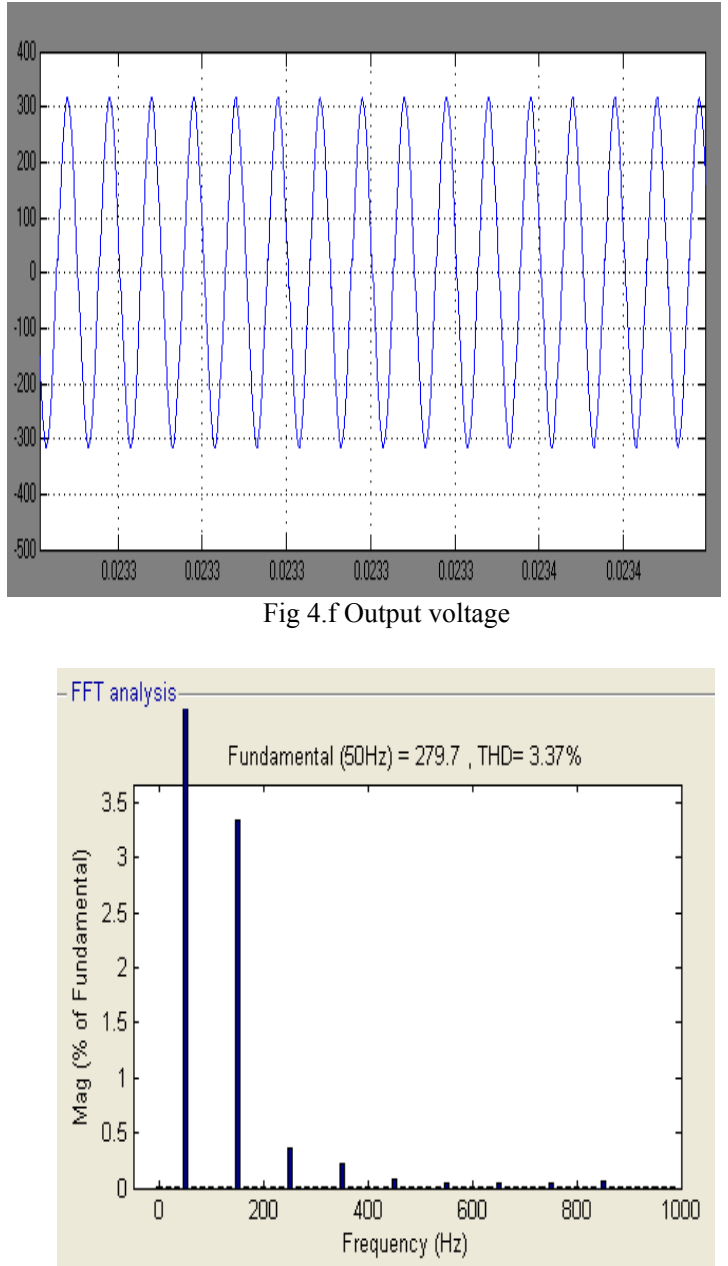

Fig 4.g FFT Analysis for output voltage

The pulse given to the second switch is shifted by 180 Degree with respect to the pulse of Switch 1.Voltage across M1 is shown in Fig 4b. Voltage across M2 is shown in Fig 4e.Voltage across the inverter is shown in Fig 4f.It can be seen that the output voltage is almost sine wave and the spectrum for the output is shown in Fig 4g. The THD value is $3.3 \%$.

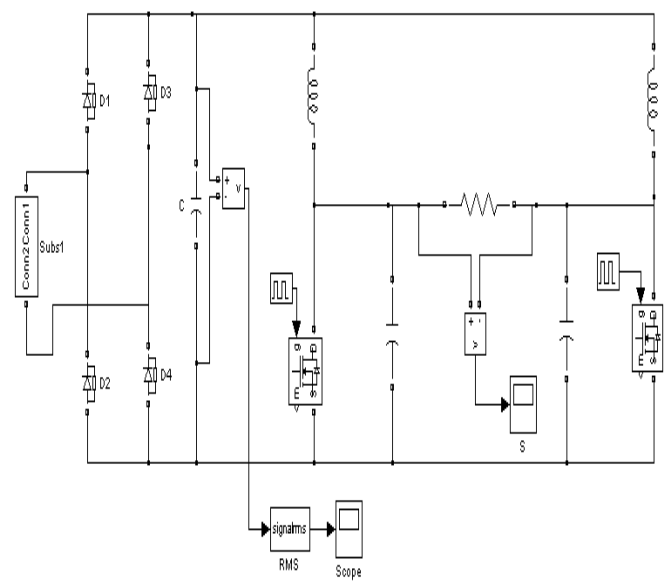

Fig.5.a. Open loop circuit model 


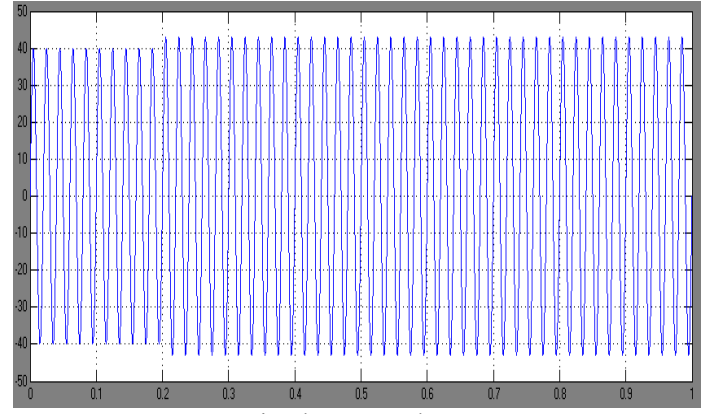

Fig5.b Input voltage

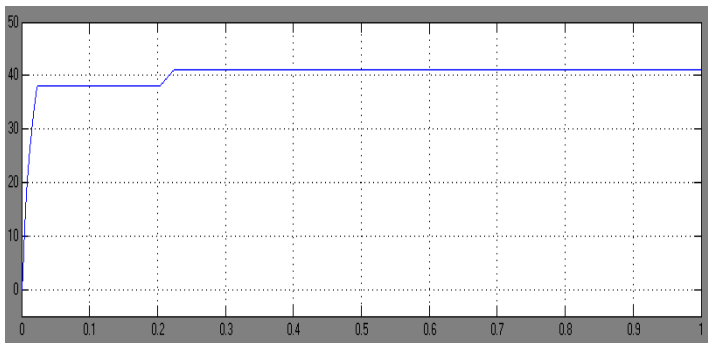

Fig 5.c Rectifier voltage

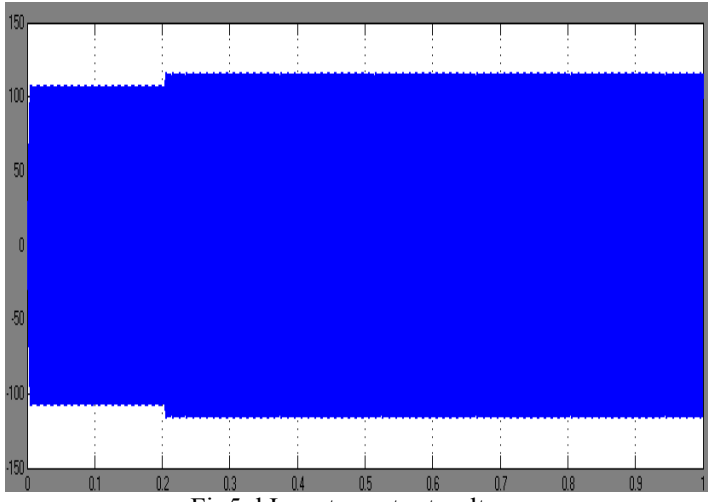

Fig5.d Inverter output voltage

Simulink model of open loop system is shown in Fig 5a.The low frequency AC input voltage is converted into DC using an uncontrolled rectifier. The output of the rectifier is converted into high frequency $\mathrm{AC}$ using Class $\mathrm{E}$ inverter. A step disturbance is applied at the input as shown in Fig 5b.The output of rectifier is shown in Fig 5.c. The Ac output voltage is shown in Fig 5.d. It can be seen that the amplitude of output increases when there is a disturbance at the input.

The closed loop circuit model is shown in Fig 6.a. The output is sensed and it is compared with the reference voltage. The error is given to a PI controller, the output of PI controller adjusts the pulse width to bring the voltage to the set value. The rectifier output is shown in Fig 6b.AC output voltage is shown in Fig 6.c.

\section{CONCLUSION}

This work has presented analysis, modeling and simulation of class $\mathrm{E}$ inverter based induction heater system. This system has advantages like low switching losses, reduced stress and increased power density. Open and closed loop models developed and they are successfully used for simulation studies. The simulation results are in line with the predictions.

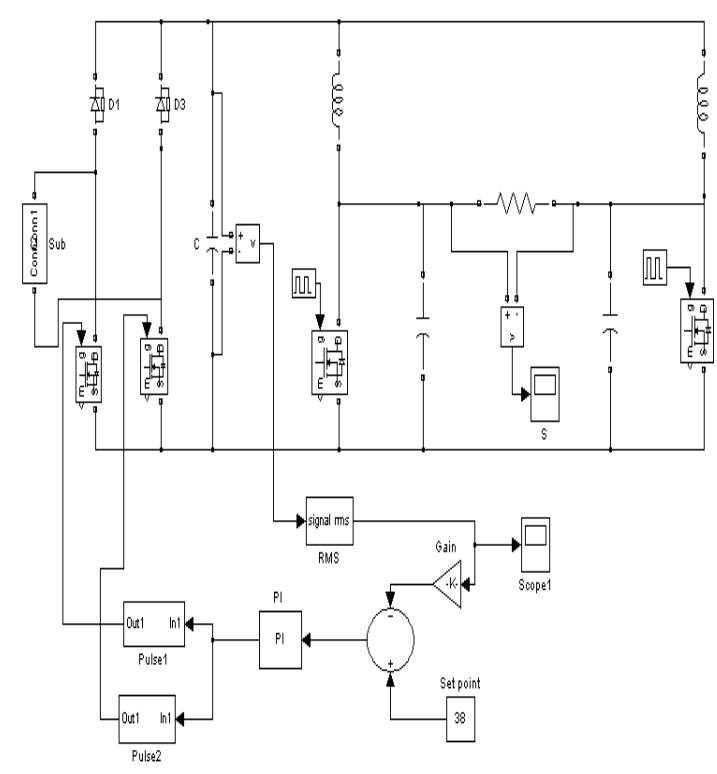

Fig.6.a. Closed loop Circuit diagram

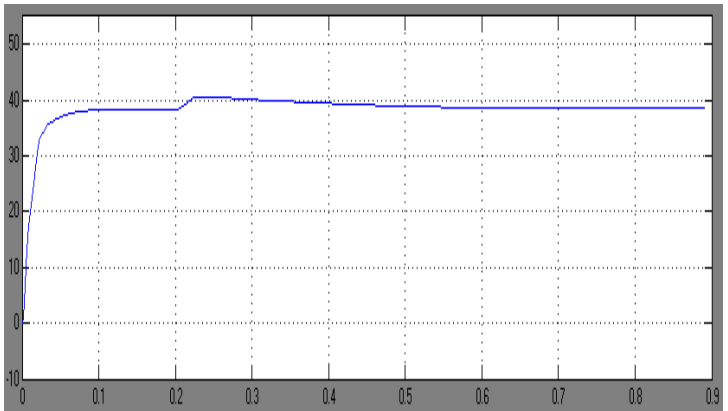

Fig.6.b.Rectifier Output Voltage

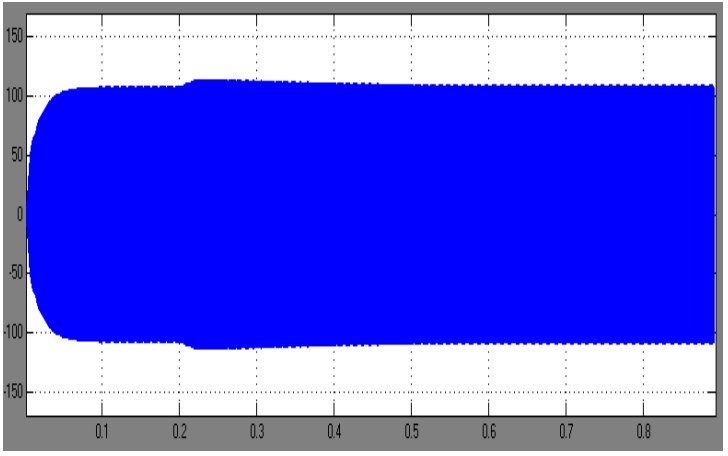

Fig.6.c. Inverter Output Voltage

\section{REFERENCES}

[1] K. Kazimierczuk, V. G. Krizhanovski, J. V. Rassokhina, and D. V. Chernov, "Class-EMOSFET tuned power oscillator design procedure," IEEE Trans. Circuits Syst I, Reg. Papers, vol. 52, no. 6, pp. 1138-1147, Jun. 2005.

[2] Y. Chen, T. J. Liang, R. L. Lin, and J. F. Chen, "A novel selfoscillating, boost-derived DC-DC converter with load regulation," IEEE Trans. Power Electron., vol. 20, no. 1, pp. 65-74, Jan. 2005.

[3] Jirasereeamornkul, M. K. Kazimierczuk, I. Boonyaroonate, and K.Chamnongthai, "Single-stage electronic ballast with Class-E rectifier as power-factor corrector," IEEE Trans. Circuits Syst I, Reg. Papers, vol. 53, no. 1, pp. 139-148, Jan. 2006.

[4] M. Rivas, R. S.Wahby, J. S. Shafran, and D. J. Perreault, "New architectures for radio frequency DC-DC power conversion," IEEE Trans. Power Electron., vol. 21, no. 2, pp. 380-393, Mar. 2006.

[5] K. Shinoda, T. Suetsugu, M. Matsuo, and S. Mori, "Analysis of phase controlled resonant dc-ac inverters with Class-E amplifiers and frequency multipliers," IEEE Trans. Ind. Electron., vol. 45, pp. 412420, Jun. 1998. 
[6] W. A. Davis and K. K. Agarwal, Radio Frequency Circuit Design. New York: Wiley, 2001, ch. 6.

[7] S. C.Wong and C. K. Tse, "Design of symmetrical Class-E power amplifiers for very low harmonic-content applications," IEEE Trans. Circuits Syst I, Reg. Papers, vol. 52, no. 8, pp. 1684-1690, Aug. 2005

[8] V. Yousefzadeh, N. Wang, Z. Popovic', and D. Maksimovic', "A digitally controlled DC/DC converter for an RF power amplifier," IEEETrans. Power Electron., vol. 21, no. 1, pp. 164-172, Jan. 2006

[9] T. Suetsugu and M. K. Kazimierczuk, "Design procedure of ClassAmplifier for off-nominal operation at 50\% duty ration," IEEE Trans.Circuits Syst I, Reg. Papers, vol. 53, no. 7, pp. 1468-1476, Jul. 2006.

[10] S. Pajic', N. Wang, P. M. Watson, T. K. Quach, and Z. Popovic','Xband two-stage high-efficiency switched-mode power amplifiers,"IEEE Trans. Microw. Theory Tech., vol. 53, no. 9, pp. 2899-2907,Sep. 2005.

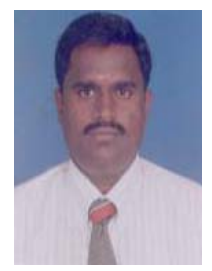

S.Arumugam has obtained his B.E degree from Bangalore University, Bangalore in the year 1999. He obtained his M.E degree from Sathyabama University, Chennai in the year 2005 . He is presently a research scholar at Bharath University, Chennai. He is working in the area of Resonant inverter fed Induction Heating.

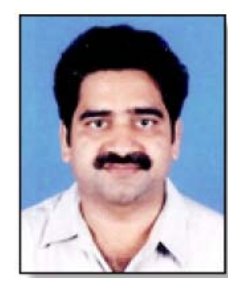

S.Ramareddy is Professor of Electrical Department, Jerusalem Engineering College, Chennai. $\mathrm{He}$ obtained his D.E.E from S.M.V.M Polytechnic, Tanuku, A.P. A.M.I.E in Electrical Engg from institution of Engineers (India), M.E in Power System from Anna University. He received Ph.D degree in the area of Resonant Converters from College of Engineering, Anna University, Chennai. $\mathrm{He}$ has published over 20 Technical papers in National and International Conference proceeding/Journals. He has secured A.M.I.E Institution Gold medal for obtaining higher marks. He has secured AIMO best project award. He has worked in Tata Consulting Engineers, Bangalore and Anna University, Chennai. His research interest is in the area of resonant converter, VLSI and Solid State drives. He is a life member of Institution of Engineers(India), Indian Society for India and Society of Power Engineers. He is a fellow of Institution of Electronics and telecommunication Engineers (India). He has published books on Power Electronics and Solid State circuits.

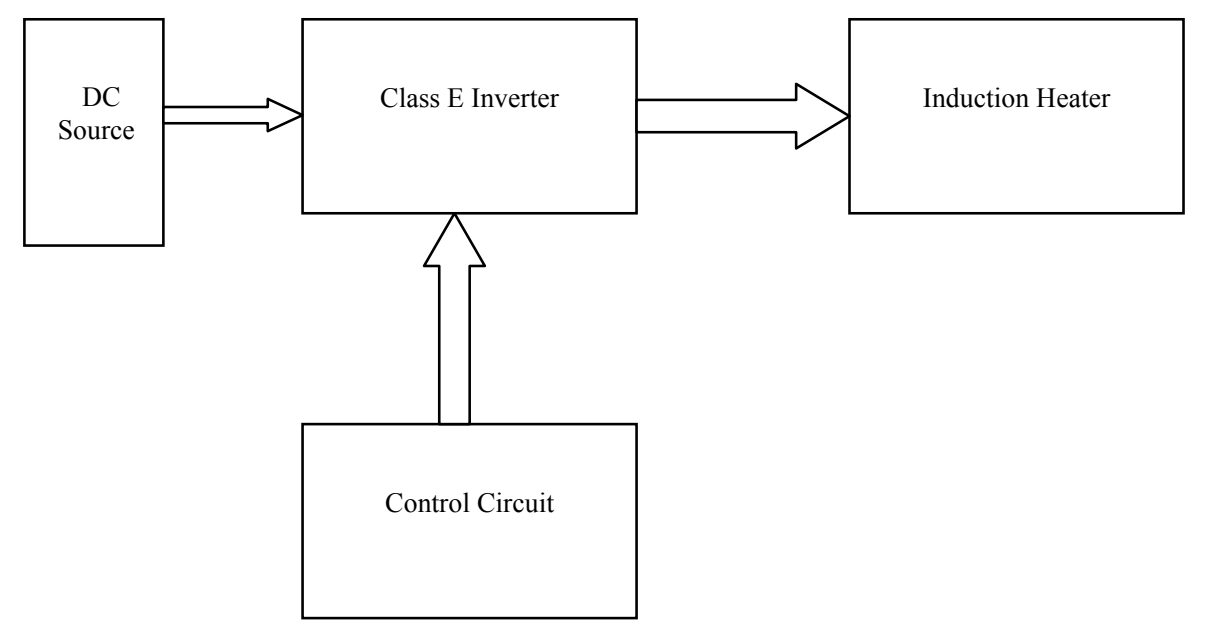

Fig. 1 Block Diagram 